Journal of

Applied Ecology

2000, 37,

914-925

\title{
Biological control using sterilizing viruses: host suppression and competition between viruses in non- spatial models
}

\author{
G.M. HOOD*, P. CHESSON† and R.P. PECH* \\ *Pest Animal Control Co-operative Research Centre, c/-CSIRO Wildlife and Ecology, PO Box 284 Canberra \\ ACT, Australia 2601; and $\dagger$ Section of Evolution and Ecology, Storer Hall, University of California, Davis, CA \\ 95616, USA
}

\begin{abstract}
Summary
1. Research is currently underway to develop genetically engineered viruses that can sterilize pest animals. The technique, known as viral-vectored immunocontraception (VVIC), promises to control mammalian pests such as the European rabbit, the house mouse and domestic cats.

2. Using host-parasite models we explored the degree of control of the host population that can be attained when hosts that recover from infection become permanently infertile. The models assume some demographic compensation for reduced fertility in the host population, and are tailored to address issues raised by the use of the myxoma virus as an agent to sterilize rabbits. A 'pay-off' function is developed, which defines the degree to which host density is suppressed by a sterilizing agent.
\end{abstract}

3. The results show that sterilizing viruses can reduce host abundance, and that hosts with low birth rates and moderate mortality rates are the best targets for VVIC. High transmissibility increases the pay-off from VVIC, but because virulent parasites kill most of the hosts that they infect, the pay-off is highest if benign parasites are used as the vector of contraceptive antigens. We argue that appropriate pay-off functions should be developed as a basis for research and development on genetically modified organisms.

4. The host-parasite models are extended to include a competing strain of virus that does not sterilize the host. We analysed these models using a general approach to the analysis of competition, which has not often been applied to epidemiological models. The extended model shows that host sterilization per se does not affect the competitive ability of viruses, a result that applies to a broad class of models in which the per capita growth rates of competing parasites are linear functions of limiting competitive factors.

Key-words: host-parasite model, immunocontraception, mammal pests, myxomatosis.

Journal of Applied Ecology (2000) 37, 914-925

\section{Introduction}

Introduced vertebrates are serious threats to biodiversity in Australia (Myers et al. 1994; Williams et al. 1995), New Zealand (Coleman 1993; Cowan \& Tyndale-Biscoe 1997) and on many islands (Atkinson 1989; Courchamp \& Sugihara 1999). The European rabbit Oryctolagus cuniculus L., for example,
Correspondence: G. M. Hood (fax 6126242 1505; e-mail greg.hood@dwe.csiro.au). is a serious threat to biodiversity and agriculture in Australia (Myers et al. 1994; Williams et al. 1995; Cowan \& Tyndale-Biscoe 1997), despite considerable success in reducing the abundance of the species using myxomatosis (Fenner \& Ross 1994) and, more recently, the calicivirus responsible for rabbit haemorrhagic disease (Cooke 1997). Research is therefore underway to develop new techniques to control rabbits and other vertebrate pests.

One research programme aims to develop recombinant sterilizing viruses (Tyndale-Biscoe 1991, 
1994; McCallum 1996; Chambers, Singleton \& Hood 1997) that, along with their normal complement of nucleic acids, will contain nucleotide sequences encoding reproductive proteins of the host (Holland \& Jackson 1994; Muller, Warren \& Evans 1997). Following natural infection with these 'immunocontraceptive' viruses, the host will develop antibodies against its own reproductive tissues, hopefully rendering the host permanently infertile. The process by which sterilizing antigens are delivered using viruses is known as viral-vectored immunocontraception (VVIC).

Before regulatory approval is granted for the release of immunocontraceptive viruses, their efficacy and safety as biological control agents must be established at several spatial scales. Efficacy, for example, must be demonstrated at three broad scales: at the scale of a single host the virus must be capable of generating a sterilizing immune response after natural infection; at the scale of a small population, the virus must infect and sterilize a sufficient number of hosts and must also be capable of persisting within that population; at still larger scales, the virus must generate enough secondary infections to ensure that it spreads among populations.

Determining efficacy in individuals is a laboratory task, but at larger scales it is not possible to assess efficacy without some form of field release. We have therefore developed mathematical models to address questions of the efficacy and persistence of immunocontraceptive viruses at the scale of a small population. A subsequent paper addresses issues raised at still larger scales (G. M. Hood, unpublished data). Host-parasite models have already been developed to study VVIC in brush-tailed possums Trichosurus vulpecula in New Zealand (Barlow 1994, 1997), and domestic cats on islands (Courchamp \& Sugihara 1999; Courchamp \& Cornell 2000), and Anderson \& May (1981) have developed generic models to study the effect of sterilization of insects by protozoan parasites. In each case, however, the sterilizing agent was assumed to cause sterility in hosts that remain infectious for their lifetime. This is an appropriate formulation for persistent infections of vertebrate hosts, such as occurs in the herpesviridae (Roizman 1990). But for VVIC in rabbits, the proposed vector of immunocontraceptive antigens is the myxoma virus (Holland \& Jackson 1994). Although this virus causes disease that can last for several weeks (Fenner \& Ross 1994), the intention is that those rabbits that recover from the infection will be sterilized. Hence, the dynamics of the recovered portion of the population must be considered in the analysis. The models presented here assume a relatively brief period of infection followed by life-long immunity and sterilization of recovered hosts. This is an appropriate formulation for VVIC using the myxoma virus or other viruses that provoke a strong immune response after acute, or subacute, infections in their mammalian host.

The first model developed here is a conventional host-parasite system with no age or stage structure. Beginning with Anderson \& May (1979), many similar models have been developed to study the interaction of population dynamics and epidemiology (Busenberg \& Hadeler 1990; Mena-Lorca \& Hethcote 1992; Zhou \& Hethcote 1994; Zhou 1995; Cooke \& Vandendriessche 1996), and further developments have been made in understanding how complications such as stage-structure (Briggs \& Godfray 1995) and seasonality (Briggs \& Godfray 1996) affect interactions between host and parasite. The first model is developed to examine how the interplay of demographic and epidemiological parameters affects establishment and persistence of the virus, and the degree to which host density is suppressed. We also consider how the degree of 'host suppression' changes if the host is sterilized immediately after infection.

The second model is an extension of the first to consider competition between sterilizing and nonsterilizing virus strains. In the case of the myxoma virus, this competition occurs between but not within hosts, because rabbits mount a completely cross-protective immunological response to the myxoma virus, meaning that after infection with one strain, any rabbit that survives is immune from infection with other myxoma viruses for life (Fenner \& Ross 1994). Competition between viruses is therefore examined here in an extension of the first model to include sterilizing and non-sterilizing virus strains, which compete for susceptible hosts.

In each model a proportion of the hosts is rendered infertile after infection with the sterilizing virus. This proportion represents the probability of success of VVIC in an individual infected host. For biological control, however, success is measured at the population level, and the purpose of the models presented here is to assess how success at the level of the individual translates to that of a population.

\section{Model structure and assumptions: Model I}

For host population dynamics, we will use a form of logistic population growth with density-dependence in the birth rate but not the death rate, as follows:

$\frac{\mathrm{d} x}{\mathrm{~d} t}=b x\left(1-\frac{x}{K^{\prime}}\right)-m x$ eqn 1

Here $x$ is the number of susceptible hosts, $b$ is the maximum per capita birth rate, $m$ is the densityindependent mortality rate, and $K^{\prime}$ is the number of hosts at which the birth rate is zero (if, for example, the availability of breeding territories limits the birth rate then $K^{\prime}$ is the number of suitable territories). Although $b$ is called the 'birth' rate, it can 
Biological control using sterilizing viruses

also be viewed as a recruitment rate into the adult population that factors in density-dependent mortality of juveniles. Equation 1, and all the equations that follow, would then describe the dynamics of the adult population only.

To simplify the algebra, we will describe the dynamics of host density, where density is expressed as a proportion of the population size at which reproduction stops, $K^{\prime}$. Equation 1 can then be written:

$\frac{\mathrm{d} X}{\mathrm{~d} t}=b x(1-X)-m X$

eqn 2

where $X=x / K^{\prime}$. Setting $\mathrm{d} X / \mathrm{d} t=0$ shows that the carrying capacity for this model is $K=1-m / b$.

If disease is introduced into a well-mixed population, new infections will arise at the rate $\beta X Y$, where $Y$ is the density of infected hosts and $\beta$ is a constant representing the rate at which infectious contacts occur. Assuming that all host types contribute equally to competition, and that a proportion, $\gamma$, of the recovered hosts, $Z$, are sterile, the dynamics of the susceptible population are:

$\frac{\mathrm{d} X}{\mathrm{~d} t}=b[X+Y+Z(1-\gamma)][1-(X+Y+Z)]$

$-(\beta Y+m) X$

eqn 3

which is a modification of the logistic dynamics of equation 2. The first term on the right-hand side of this equation says that all host types $(X+Y+Z)$ contribute to density-dependence in the host growth rate and that $\gamma Z$ of the recovered hosts do not breed; the second term is the rate at which susceptible hosts are lost via new infections, $\beta Y$, and natural deaths, $m$. Although diseases like myxomatosis are debilitating in mammalian hosts and can affect reproduction in males (Poole 1960; Fountain et al. 1997), the model assumes that female fertility is unaffected by infection or reduced male fertility.

If there is no latent period, and infected hosts have additional mortality $\alpha$ and recover at rate $v$, the rate of change in their density is:

$\frac{\mathrm{d} Y}{\mathrm{~d} t}=\beta X Y-(\alpha+m+v) Y$.

eqn 4

We assume further that recovered hosts are permanently immune from further infection, and have the same background mortality rate as susceptible hosts. The rate of change in their density $Z$ is then given by the expression:

$\frac{\mathrm{d} Z}{\mathrm{~d} t}=v Y-m Z$

eqn 5

(C) 2000 British Ecological Society Journal of Applied Ecology, 37, 914-925
Together, equations 3-5 (Model I) specify a modification of the disease-host models of Anderson \& May (1979) to include density-dependent growth of the host and sterilization of some recovered hosts.

\section{Preliminary analysis of the model}

To understand the model, first note that summing equations 3-5 produces an expression for the total rate of change of the host population:

$\frac{\mathrm{d} N}{\mathrm{~d} t}=b(N-\gamma Z)(1-N)-\alpha Y-m N, \quad$ eqn 6

where $N=X+Y+Z$ is the population density. Comparison of this equation with equation 3 shows that the effect of the virus on the host population is twofold: the birth rate is reduced by a proportion $\gamma Z / N$ because sterile recovered hosts do not breed; and, if the virus is also virulent (i.e. $\alpha>0$ ), the death rate is increased by $\alpha Y$ because of the additional mortality of infected hosts.

If a small number of infected hosts is introduced to a fully susceptible population, solving equation 4 for $\mathrm{d} Y / \mathrm{d} t=0$ defines a threshold density of susceptible hosts, $X_{\mathrm{T}}$, beyond which the infection will grow, and below which the infection will die out:

$X_{\mathrm{T}}=\frac{\alpha+m+v}{\beta}$.

eqn 7

Persistence of parasites in simple epidemiological models is governed by the basic reproductive rate, $R_{0}$, which is the number of secondary infections that one infected host could generate if introduced into a completely susceptible population (Anderson \& May 1991; Dietz 1995). The endemic state of the virus can be attained only if $R_{0}>1$, which means that each infected host must infect (on average) more than one new host before the currently infected host dies. For this to be so, the carrying capacity (the equilibrium achieved with no infection) must be greater than the threshold density. To calculate $R_{0}$ we note from equation 4 that infectives are lost through death or recovery at the rate $\alpha+m$ $+v$, which means that the average duration of infection in an individual is $1 /(\alpha+m+v)$ units of time. With the susceptibles at carrying capacity, an individual infective produces $\beta K$ new infectives per unit time, which implies that we must have:

$K>\frac{\alpha+m+v}{\beta}$,

eqn 8

and:

$R_{0}=\frac{\beta}{\alpha+m+v}$.

eqn 9

Condition 9 is a requirement for successful invasion of the virus into the host population. A local stability analysis presented in Hood (2000) and simulations (e.g. Fig. 2) show that, when and only when condition 8 is satisfied and $b \geq m$, there is a unique, locally stable equilibrium between the virus and host populations with the virus at positive density. 
G.M. Hood,

$P$. Chesson \&

R.P. Pech

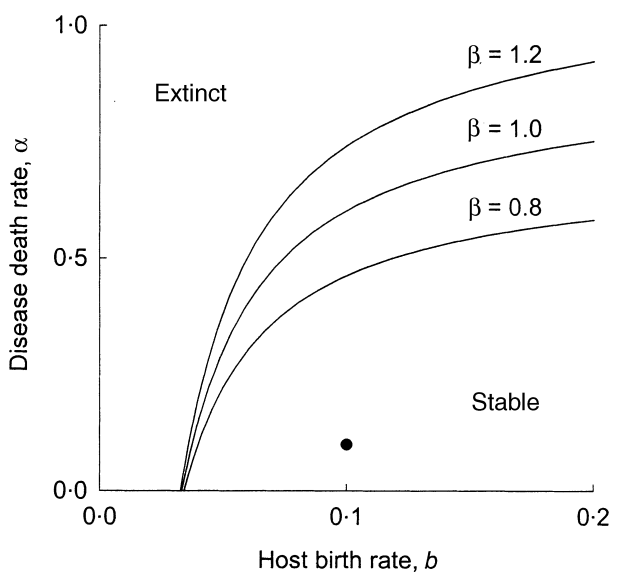

Fig. 1. Model I: stability portrait as a function of the host birth rate, $b$, and the disease-induced mortality rate, $\alpha$, for three different values of the transmissibility, $\beta$. Each line divides the parameter space into regions where the virus can and cannot persist, according to equation 9 with $R_{0}=$ 1. Other parameters $K^{\prime}=1, m=0.03, v=0.067$ and $\gamma=0$. Default values of $b$ and $\alpha$ are shown as a filled circle, assumes $\beta=1$.

Equating $R_{0}$ to one in equation 9 gives a relationship that divides the parameter space into regions where the infection can and cannot persist: $\alpha=\beta(1$ $-m / b)-(m+v)$. A plot of the relationship (Fig. 1) confirms the intuition that the virus can only persist if the host birth rate is sufficiently high and the virus does not kill hosts too quickly. Notice that $\alpha$ is linear in $m$ and $\beta$, and so different values of these parameters change the slope of the relationship between $\alpha$ and $b$, as shown in the figure using different values of the transmissibility, $\beta$.

Within the region of persistence, equilibrium host densities can be obtained by setting each of equations 3-5 to zero and solving the resulting set of simultaneous equations. At equilibrium $\mathrm{d} X / \mathrm{d} t=0$, and therefore susceptible hosts are regulated to the threshold density given by equation 7. Similarly, equation 5 gives the equilibrium density of recovered hosts as $v Y^{*} / m$, where $Y^{*}$ is the equilibrium density of infectives. Unfortunately, the density of infectives at the equilibrium is a solution to the following quadratic, which can be solved but is not easily interpreted:

$$
\begin{aligned}
& -\frac{1}{m^{2}} b(m+v)[m+(1-\gamma) v] Y^{* 2} \\
& +\left\{-d+\frac{1}{\beta m} b[-2 m d+\beta m-v d\right. \\
& -v(1-\gamma)(d-\beta)]\} Y^{*} \\
& +\frac{d}{\beta^{2}}[-b d+\beta(b-m)]=0,
\end{aligned}
$$

where $d=\alpha+m+v$. The total density of the host population, $N^{*}=X^{*}+Y^{*}+Z^{*}$, is an equally complicated function of the parameters, and therefore the degree to which host density is suppressed is explored in the following by graphical means.

\section{Default parameter values}

The effect of the parameters on host density was explored by varying each parameter in turn with the remaining parameters held at their default values (Table 1). The default values were chosen to capture the chief features of the rabbit-myxoma virus interaction (the main target of the VVIC programme in Australia). Because the model is designed to address qualitative, rather than quantitative, issues, the parameter estimates need not be exact, but the relative time scales of demographic and infection processes are important determinants of the behaviour of host-parasite models (Onstad 1992), and so it is important that parameters are within an appropriate range. The default values are briefly justified in this section and the effect of variation around these values is explored in the next section.

Rabbit demography varies greatly throughout Australasia (Gilbert et al. 1987), and so it is difficult to set representative values for the demographic parameters $b$ and $m$. Assuming the survival prob-
Table 1. Default parameters of the non-spatial model. Rate parameters $(\alpha, v, \beta, b$ and $m)$ are expressed in units of week ${ }^{-1}$; $R_{0}$ and $K$ are expressed as a proportion of the density at which the density-dependent birth rate goes to zero, $K^{\prime}$

\begin{tabular}{lll}
\hline Parameter & Default value & Explanation \\
\hline$\alpha$ & $0 \cdot 1$ & Rate of disease-induced deaths in infected hosts \\
$\nu$ & $0 \cdot 067$ & Recovery rate of infected hosts \\
$\beta$ & 1 & Transmission rate \\
$\gamma$ & 0 & Proportion of recovered hosts sterilized \\
$b$ & $0 \cdot 1$ & Low-density birth rate \\
$m$ & 0.03 & Mortality rate (independent of density) \\
$R_{0}$ & 5.08 & Dependent parameter-basic reproductive rate \\
$K$ & $0 \cdot 7$ & Dependent parameter-carrying capacity \\
\hline
\end{tabular}


918

Biological control using sterilizing viruses
(C) 2000 British Ecological Society Journal of Applied Ecology, 37, 914-925 ability, averaged over all age classes, is 0.2 year $^{-1}$, the mortality rate is $m=-\ln (0.2) / 52 \approx 0.03$ week $^{-1}$, which is at the higher end of the range for adult rabbits in Australia but lower than typical values for kittens (Gilbert et al. 1987). It is also the same as the estimate of Barlow \& Kean (1998).

Birth rates of rabbits can be very high. Adult females, for example, are capable of producing seven litters per year, with each litter averaging around five to six kittens and each kitten capable of breeding by 3-5 months of age (Mykytowycz \& Fullagar 1973). We assume $b=0.1$ week $^{-1}$, and $m=$ 0.03 , which yields an annual instantaneous growth rate in the absence of density-dependence of $r=3 \cdot 6$. This is larger than the values presented by Hone (1999) and those assumed in other models of the population dynamics of rabbits (Gilbert et al. 1987; Smith \& Trout 1994; Barlow \& Kean 1998) but similar to the maximum rate of increase estimated by Pech \& Hood (1998) of $r_{\max }=3.44$. Here, our estimate excludes one of the major agents of mortality, myxomatosis, and it is therefore appropriate that it is set near $r_{\max }$. A population with this growth rate would grow from a density of 0.01 to about $0.23 K^{\prime}$ within a year, and be close to the carrying capacity $\left(K=1-m / b=0 \cdot 7 K^{\prime}\right)$ within 2 years.

Epidemiological parameters are equally difficult to set because virulence and transmissibility of myxomatosis depend on the strain of virus and the genetic resistance of rabbits. In wild rabbits, for example, case-fatality rates can be as low as $30 \%$ or as high as 100\% (Fenner \& Ross 1994; P. J. Kerr, unpublished data), and the duration of the infectious period ranges from less than 1 week to more than 7 weeks (Parer et al. 1994; P. J. Kerr, unpublished data). For the default parameter set, we assume a relatively long infectious period of 6 weeks, and so $1 /(\alpha+v) \approx 6$ (ignoring natural mortality, $m)$. If $60 \%$ of infected hosts die, we have $\alpha /(\alpha$ $+v)=0.6$, and $v /(\alpha+v)=0.4$, and hence $\alpha=0.1$ and $v \approx 0.067$.

There have been several attempts to estimate transmission rates of myxomatosis (Saunders 1980; Seymour 1992), but because insect vectors spread the disease among rabbits, transmission rates depend on seasonal changes in vector activity (Edmonds, Shepherd \& Nolan 1978; Parer \& Korn 1989; Ross et al. 1989). In this simple model, we assume that myxomatosis is spread by the European rabbit flea Spilopsyllus cuniculi (Dale), which often produces epidemics that last several months (Kerr et al. 1998). For a sensible model, the value of $\beta$ should be higher than the birth rate so that disease transmission is more rapid than reproduction. We chose $\beta=1$, which is an order of magnitude larger than the default birth rate. In a naive population at the carrying capacity, this value of $\beta$ generates an epidemic that lasts for about 6 months, after which there are oscillations lasting about 18 months before

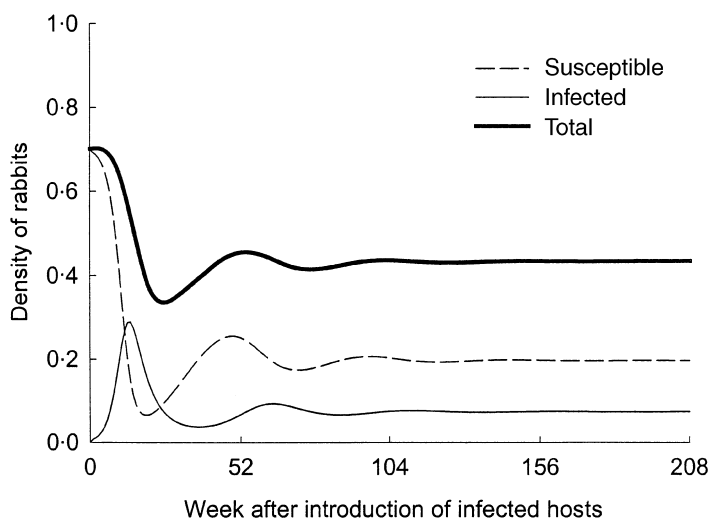

Fig. 2. Model I: trajectory following introduction of infected hosts (at density $=0.001$ ) to a fully susceptible population at the carrying capacity with the default parameter set.

the population settles to equilibrium densities (Fig. 2).

\section{Effect of demographic and infection parameters on equilibrium densities}

For any virus used for biological control, there are at least two important questions that must be addressed: first, can the virus persist in the host population; and secondly, by how much is the host population suppressed? If a sterilizing agent is to be developed using an extant virus, a third question arises: by how much is host density reduced in relation to current densities (i.e. those attained in the presence of field strains of the virus).

The first question is easily answered: persistence conditions for the virus are obtained by solving equation 9 for $R_{0}=1$. Persistence is enhanced by high birth rates and high transmissibility, but compromised by rapid loss of infected hosts $(\alpha+v)$ or an excessive natural death rate. For any pair of parameters it is simple to construct stability portraits like that shown as Fig. 1.

By inspection of equation 6 , it is clear that in a closed population, sterilization of the host compromises the production of new susceptible hosts. However, the intuitive notion that this should jeopardize the persistence of the virus is not realized, because condition 8 says that persistence is independent of the sterilization rate, $\gamma$. This result occurs because invasion and persistence of the virus depends on the longevity of infected hosts (i.e. on $\alpha, m$ and $v$ ) but is independent of any effect of the virus on host reproduction. Thus, the answer to the first biological control question (can a sterilizing virus persist in the host population?) is that the persistence conditions for a sterilizing virus are precisely the same as those of a non-sterilizing virus. Simply put, sterilization 
919

G.M.Hood,

P. Chesson \&

R.P. Pech
(C) 2000 British Ecological Society Journal of Applied Ecology, 37, 914-925

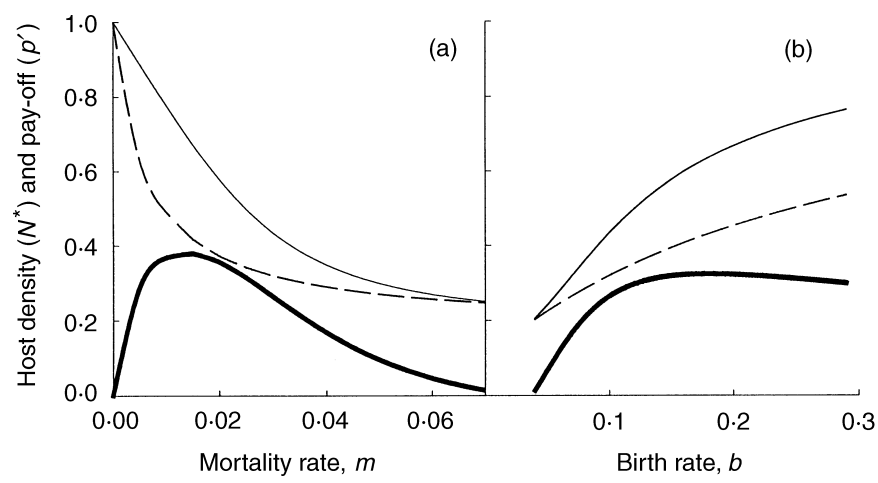

Fig. 3. Relationship of equilibrium host densities in Model I to host demography. Fine lines shows the host density $N^{*}$ for non-sterilizing (solid line) and sterilizing (dashed line). Thick lines show the pay-off, $p^{\prime}=1-N^{*} \gamma / N^{*}{ }_{0}$. Other parameters set to default values.

has a negligible effect on the availability of susceptible hosts while the number of infected hosts is small, and therefore does not affect invasion and persistence of the host. Sterilization only has an appreciable effect on host availability when the sterilizing virus is abundant.

To determine the degree of host suppression by a sterilizing virus, we follow Beddington, Free \& Lawton (1978) in defining host suppression, $q$, as the degree to which the host population is reduced below the carrying capacity. That is $q=N^{*} / K$, where $0 \leq q \leq 1$ and $N^{*}$ is the equilibrium host density. For presentation of the results, it is useful to define the 'pay-off' realized by the introduction of a virus as $p=1-N^{*} / K$. Furthermore, if an endemic virus is engineered, we assume that the host population is already regulated to some degree by field strains of the virus. The appropriate measure of the pay-off is then $p^{\prime}=1-N^{*} \gamma / N^{*}{ }_{0}$, which is one minus the host density realized in the presence of a sterilizing virus divided by the density realized when a non-sterilizing virus is present in the population.

Figure 3 shows the dependence of host densities on the demographic parameters for non-sterilizing $(\gamma=0)$ and sterilizing $(\gamma \rightarrow 1)$ viruses. Notice that the pay-off is optimal when the host has a moderate mortality rate. This result occurs because the sterilizing virus reduces host density at moderate values of $m$ but dies out at extreme values. That is, as $m \rightarrow 0$ recovered hosts accumulate in the population and both sterilizing and non-sterilizing viruses die out; while as $m \rightarrow \propto$ natural deaths occur too quickly to allow the infection to establish. The pay-off is also maximized at moderate birth rates, because as $b \rightarrow$ 0 no new susceptible hosts are produced and so the infection dies out; conversely, as $b \rightarrow \propto$ the rate at which new hosts are produced outstrips the infection rate, so that neither sterilizing nor non-sterilizing viruses affect the equilibrium host density. Hence, the pay-off is greatest at moderate birth rates.

For non-sterilizing viruses, host density is minimized when the virulence, $\alpha$, is moderate (Fig. 4a), mirroring the findings of Anderson \& May (1979, 1981). For a sterilizing virus, however, host density increases with $\alpha$, and the pay-off therefore decreases. The increase in host density with $\alpha$ occurs because the infection dies out as $\alpha \rightarrow \propto$ (because $R_{0} \rightarrow 0$, condition 9), while as $\alpha \rightarrow 0$, a greater proportion of

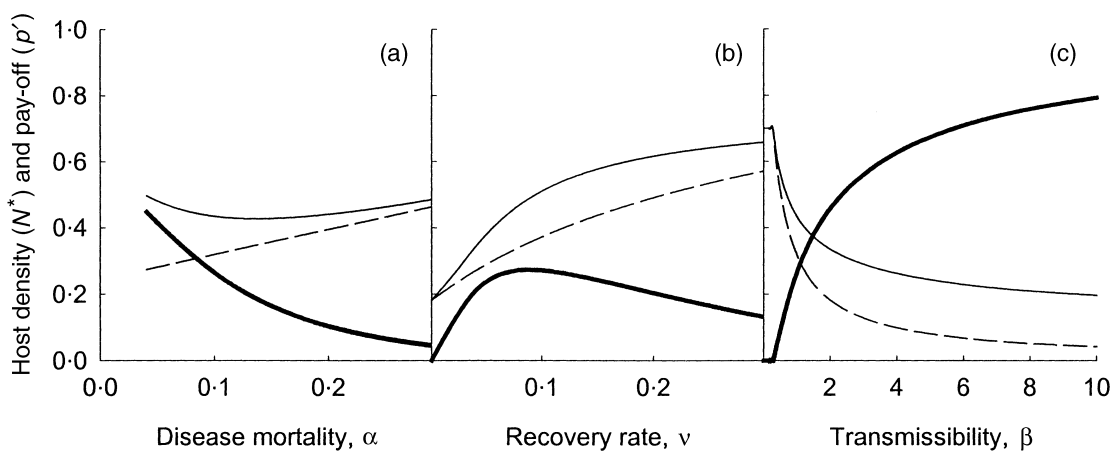

Fig. 4. Relationship of equilibrium host densities in Model I to epidemiological parameters. Fine lines shows the host density $N^{*}$ for non-sterilizing (solid line) and sterilizing (dashed line). Thick lines show the pay-off, $p^{\prime}=1-N^{*} \gamma / N_{0}^{*}$. Other parameters set to default values. 
Biological control using sterilizing viruses
(C) 2000 British Ecological Society Journal of Applied Ecology, 37, 914-925 the infected hosts are sterilized, and so host density declines.

Because suppression of the host population by the sterilizing virus depends on preventing reproduction by recovered hosts, the pay-off increases with the recovery rate, $v$, but only to a point. As $v \rightarrow \propto$ the infection can no longer be maintained (condition 9), while as $v \rightarrow 0$ the sterilizing virus has no effect on the host. Hence the pay-off is maximized at intermediate values of $v$ (Fig. $4 \mathrm{~b}$ ).

An increase in transmissibility increases the payoff (Fig. 4c), a result that occurs because high transmissibility can overcome the inhibition of the rate of new infections that occurs in the presence of a sterilizing virus.

A more intuitive understanding of the role of the epidemiological parameters in determining host abundance can be obtained by analysing the pay-off in terms of case fatality rates and survival times, the quantities most often measured in epidemiological studies (Parer et al. 1994). Ignoring natural mortality (i.e. assuming $m$ is small), we define the case fatality, $C F=\alpha /(\alpha+v)$, as the proportion of the infected hosts that die as a result of the infection. The survival time (once again, ignoring natural mortality) is $\delta=1 /(\alpha+v)$. Note that, if the case fatality is high, few hosts recover and the proportion of infected hosts that are sterilized will be low, regardless of the sterilization rate, $\gamma$. We should therefore expect that the pay-off due to engineering a virulent virus $(C F \rightarrow 1)$ should be less than could be expected with a benign virus $(C F \rightarrow 0)$.

Figure 5a summarizes the pay-off for a sterilizing virus as a function of the case fatality and the duration of the infectious period. As predicted, the payoff from VVIC is highest for benign sterilizing viruses $(v \gg \alpha)$, but the maximum pay-off is achieved when the infectious period is of moderate duration. The decline in the pay-off when the infectious period is prolonged (greater than $\sim 10$ weeks) occurs because it takes a long time before infected hosts recover and become sterile. In fact, for viruses with long periods of infection, models such as those developed by Barlow (1994) are more appropriate than Model I, because the sterilizing response depends on immunological events triggered well before recovery from infection occurs. If the immunocontraceptive response is instantaneous, equation 3 of Model I could be modified as follows to allow for sterilization of infected hosts:

$\frac{\mathrm{d} X}{\mathrm{~d} t}=b[X+Y(1-\gamma)+Z(1-\gamma)]$

$[1-(X+Y+Z)]-(\beta Y+m) X$

eqn 11

With this modification the equilibrium densities of each host type can be calculated, but the resulting expressions are not easily interpreted (Hood 1999).
The pay-off, however, is easily calculated: it is everywhere higher than predicted by Model I (Fig. 5b) and does not decline as the duration of infection increases. Notice that both models predict similar outcomes if the duration of the infectious period is short.

We conclude from this analysis that benign viruses (those with a long duration of infection and a low case fatality) will generally be the best agents for delivery of immunocontraceptive antigens. This conclusion, however, ought to be tempered by consideration of any relationship between virulence, transmissibility and the duration of the infectious period. In the case of the myxoma virus, the probability of transmission of the disease depends directly on the density of virus in skin lesions that are probed by mosquitoes and other insect vectors (Fenner \& Ratcliffe 1965). The development of virus-laden skin lesions and the duration of infection

(a)

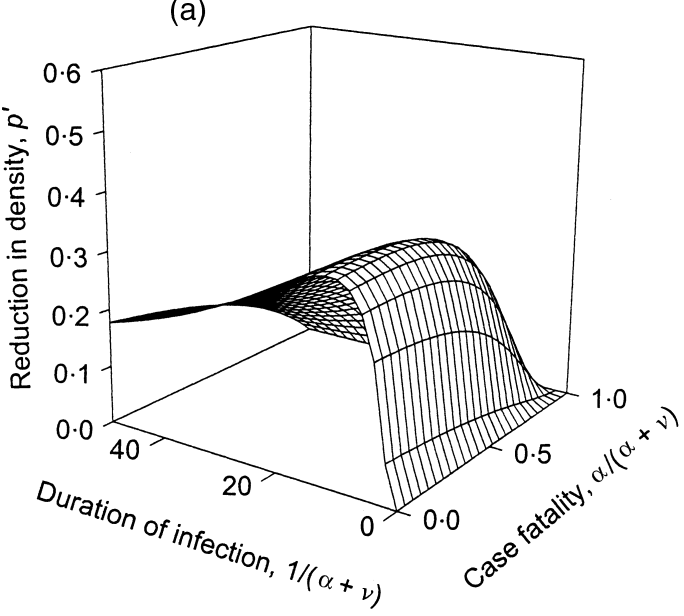

(b)

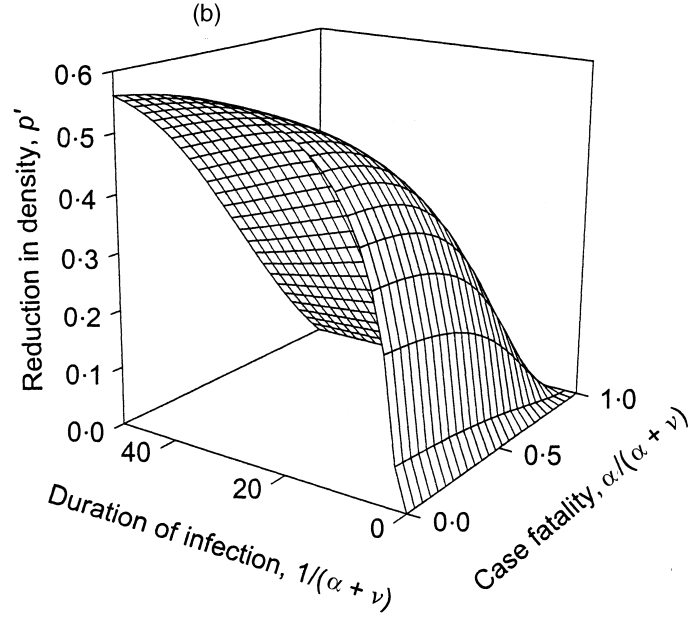

Fig. 5. The pay-off from immunocontraception as a function of the duration of the infectious period and the case fatality rate, (a) for Model I and (b) for the modified model which allows for sterilization of infected hosts (see equation 11). The proportion of the recovered hosts sterilized by the sterilizing virus is $\gamma=0 \cdot 8$. 
G.M. Hood,

P. Chesson \&

R.P. Pech depend in turn on the virulence of the virus (Levin \& Pimental 1981; Anderson \& May 1982; May \& Anderson 1983; Massad 1987; Dwyer, Levin \& Buttel 1990; Nowak \& May 1994). These interactions are beyond the scope of the current work, but their implications for VVIC can be determined if the functional relationship between transmissibility and virulence is known. For example, we have also analysed a model with $\beta=k \alpha$, where $k$ is some constant. Analysis of that model (not presented here) shows that viruses of intermediate virulence yield the best pay-off.

\section{Competition between sterilizing and non- sterilizing parasites: Model II}

In Model I, even though the density of infected hosts declines with the sterilization rate, persistence of the virus is unaffected by $\gamma$. In the presence of competing viruses, however, the sterilization rate might be expected to affect the conditions for persistence.

Some models of competition between parasites (Levin \& Pimental 1981; May \& Nowak 1994; Nowak \& May 1994) assume that co-infection of the host can occur, and that one parasite (or strain of parasite) can competitively displace another parasite within the host. However, the model presented here ignores co-infection with multiple strains, which is believed to be uncommon with myxomatosis (see the discussion in Dwyer, Levin \& Buttel 1990, p. 429).

If co-infection does not occur, we can write the following model:

$$
\frac{\mathrm{d} X}{\mathrm{~d} t}=b\left(N-\gamma Z_{s}\right)(1-N)-\left(\sum_{i} \beta_{i} Y_{i}+m\right) X
$$

eqn 12

$\frac{\mathrm{d} Y_{i}}{\mathrm{~d} t}=\beta_{i} X Y_{i}-\left(a_{i}+m+v_{i}\right) Y_{i}$

$\frac{\mathrm{d} Z_{i}}{\mathrm{~d} t}=v Y_{i}-m Z_{i}$

Here $N=X+\sum_{i}\left(Y_{i}+Z_{i}\right)$ is the host population density and $i \in\{s, f\}$ where the indices denote hosts infected with, or recovered from, the sterilizing $(s)$ and non-sterilizing $(f)$ strains.

Equations 12-14 constitute Model II. In brief, equation 12 is a modification of 3 to allow for infection of susceptible hosts by either virus strain; 13 is an analogue of 4 describing the rate of change in density of hosts infected with each virus; and 14 is the rate of change in the density of recovered hosts of each strain. Notice that new infections are established only in susceptible hosts, and so we are assuming that infection with one strain precludes infection with the other strain.

One way to determine the outcome of competition in Model II is to calculate $R_{0}$ for each strain. Assuming that the strain with the highest value of $R_{0}$ excludes all others (Anderson \& May 1982; Bremerman \& Thieme 1989), a condition for competitive exclusion of the non-sterilizing strain is:

$\frac{\beta_{s} X}{\alpha_{s}+m+v_{s}}>\frac{\beta_{f} X}{\alpha_{f}+m+v_{f}}$

eqn 15

which depends neither on the sterilization rate nor the abundance of susceptibles. To compete effectively, an immunocontraceptive virus must be more transmissible, $\beta_{s}>\beta_{f}$, and/or have a longer infectious period $\left(\alpha_{s}+v_{s}\right)<\left(\alpha_{f}+v_{f}\right)$, than non-sterilizing strains, a result that has also been shown in other studies of competition amongst sterilizing parasites (Barlow 1994, 1997).

Maximization of $R_{0}$ is a valid criterion for analysing competition in simple epidemiological models, but the approach can give misleading results in structured models with non-linear dynamics (Nowak \& May 1994; Ferriere \& Gatto 1995). We therefore prefer a general approach to the analysis of competition developed by Chesson (1994; Chesson \& Huntly 1997). This approach considers the change in relative abundance of one species with respect to another. If this quantity has constant sign, then one species must eventually exclude the other. Chesson \& Huntly (1997) define the relative abundance, $A_{1,2}$, of competing species 1 and 2 (using our terminology) as the scaled difference in log population sizes at time $t$ :

$A_{1,2}=\frac{\ln \left[Y_{1}(t)\right]}{\beta_{1}}-\frac{\ln \left[Y_{2}(t)\right]}{\beta_{2}}$

eqn 16

Here, the transmission rates $\beta_{i}$ appear as scaling factors representing the magnitude of the response of each parasite to the common competitive factor, the density of susceptible hosts, $X(t)$. Although this seems an unusual measure of relative abundance, Chesson \& Huntly (1997) explain that it correctly predicts competitive exclusion in the sense that if $A_{1,2}$ increases to infinity, strain 1 excludes 2 , and if it decreases to minus infinity, 2 excludes 1 .

We can use equation 13 and the fact that the change in $\log$ abundance is $\frac{1}{Y} \cdot \frac{\mathrm{d} Y}{\mathrm{~d} t}$ to write an expression for the rate of change in relative abundance:

$\frac{\mathrm{d} A_{s, f}}{\mathrm{~d} t}=-\frac{\alpha_{s}+m+v_{s}}{\beta_{s}}+\frac{\alpha_{f}+m+v_{f}}{\beta_{f}}$ eqn 17

which has constant sign at all host densities. We have arrived at the same condition for persistence of the sterilizing virus derived using $R_{0}$, but the advantage of this approach is that we can now see Model 
922

Biological control using sterilizing viruses
C) 2000 British Ecological Society Journal of Applied Ecology, 37, 914-925
II as a special case of a broad class of models in which there is a single linear competitive factor. That is the per capita growth rate of species $i$ is of the form:

$\frac{1}{Y} \cdot \frac{\mathrm{d} Y_{i}}{\mathrm{~d} t}=\beta_{i} X+\alpha_{i}+m+v_{i}$

eqn 18

Coexistence cannot occur in a system in which there is a single linear competitive factor (Chesson \& Huntly 1997). The sterilizing virus can only persist if it satisfies inequality 15 , in which case the non-sterilizing form is competitively eliminated. The general results of Chesson \& Huntly (1997) show that this conclusion remains true in a temporally variable environment if the environment does not affect $\beta_{i}$, regardless of how much it affects the other parameters of the model. However, temporal environmental variation affecting $\beta_{i}$ makes the model non-additive in the sense of Chesson \& Huntly (1997) and gives the potential for coexistence of the strains. Coexistence in a variable environmental is also a possibility under some conditions if the linear per capita growth rate (equation 18) is replaced by a non-linear form. Various forms of spatial variation also have the potential to permit coexistence of the two strains (Chesson 1997) and is the subject of subsequent work.

\section{Discussion}

In a generic sense, the pay-off function developed here is a fundamental quantity that ought to determine the allocation of resources to research and development of genetically modified organisms. Analysis of the pay-off function can reveal surprising results. For example, intuition suggests that an agent that affects both survival and reproduction of the host would be most effective for biological control. While this is almost certainly true if a sterilizing agent is introduced into a naive population (for which the appropriate measure of the pay-off is $p=$ $\left.1-N^{*} / K\right)$, our analysis shows that if the aim is to engineer an endemic parasite (for which the pay-off is $p^{\prime}=1-N^{*} \gamma / N_{0}^{*}$ ) we should choose agents that are relatively benign, unless there are trade-offs between virulence and transmissibility.

Reduction of host density using VVIC depends on immunosterilization of recovered hosts, and so in a sense the more that recover the better the pay-off. It should be remembered here that our definition of the pay-off, $p^{\prime}$, encapsulates additional benefits due to engineering an extant parasite, and therefore factors out any benefits due to mortality of infected hosts. One practical implication is that VVIC may be a useful technique to rescue the efficacy of a conventional biological control agent that has lost impact due to declining virulence, as has apparently occurred with the myxoma virus. The expense of development, though, might not be justifiable if virulent parasites are used.

Although the pay-off decreases for extremely fecund species (Fig. 3b), the upper range of birth rates depicted in the figure is too high for most vertebrates, and so we concur with others (Stenseth 1981; Tyndale-Biscoe 1991, 1994; Hone 1992) who have concluded that fecund species are the best targets for VVIC. Recently, Hone (1999) showed that small-sized species, which are characterized by high rates of increase, $r$, would be more difficult to control than larger species, because a greater proportion of the population must be sterilized to stop growth of an increasing population. Hone's analysis, though, is not designed to address epidemiological issues, nor does it allow for density-dependent responses of the host.

Because the pay-off is small when host mortality is very low (Fig. 3a), long-lived species are not good targets for immunocontraception. This conclusion mirrors earlier work (Stenseth 1981; Hone 1992; Barlow, Kean \& Briggs 1997) showing that populations with low turnover are not good targets for control by sterilization. However, the result arises here partly because of the inhibition of the force of infection that occurs when most of the population consists of recovered-immune hosts, which have a high survival rate and do not sustain the infection. If the force of infection is not closely related to density, as is likely with some vector-borne diseases, it may still be possible to achieve good results in longlived species using immunocontraceptive agents.

Density-dependence in host demography also affects the pay-off from biological control with a sterilizing parasite. The form of density-dependence in Models I and II is motivated by studies in rabbits (Mykytowycz 1961; Mykytowycz \& Fullagar 1973), showing that females respond to social pressures at high population densities by reducing per capita reproductive output. It also makes the models relatively easy to analyse, because there are no densitydependent terms in the equations describing the rates of change of the infected and recovered parts of the population. Recently, studies of surgically sterilized rabbit populations (Williams \& Twigg 1996; Twigg et al. 2000) have shown that survival of kittens through their first summer is inversely related to the number of kittens produced in a breeding season. As suggested earlier, however, density-dependent mortality of juveniles is factored into Model I if the state variables represent adult densities: the birth rate, $b$, would then represent the recruitment rather than births per se, and only the adult mortality would be independent of density. Hence, if controlling the adults is of primary interest, Model I does not need adjustment to examine the effects of density-dependent mortality unless the virus affects recruitment to the host population, in 
G.M. Hood,

$P$. Chesson \&

R.P. Pech

(C) 2000 British

Ecological Society

Journal of Applied

Ecology, 37.

914-925 which case an age-structured model must be developed.

A 'hidden' assumption in the models is that per capita reproductive effort declines instantly and linearly with density (equation 3 ). Other forms of density-dependence may lead to somewhat different conclusions, but need to be developed for specific host-parasite systems and need to be carefully validated before quantitative predictions are made.

We have assumed that all of the processes leading to new infections can be summarized in the incidence function, $\beta X Y$, an assumption called linear transmission (Hethcote \& van den Dreissche 1991). But diseases like myxomatosis are transmitted by insect vectors, and so the incidence function encapsulates many factors, the density of vectors, the probability of being bitten by a vector, and so on, that are important for transmission of the disease but difficult to quantify. Non-linear incidence functions (that is, new infections arise at rate $\beta X^{g} Y^{h}$ where $g, h \neq 1$ ) have also been discussed in the literature (Liu, Levin \& Iwasa 1986; Liu, Hethcote \& Levin 1987). Non-linear incidence could occur in sociable hosts that cluster together when their numbers are depleted, thereby maintaining high contact rates at low density. Indeed, empirical evidence of non-linear incidence has already been noted for the myxoma virus (Saunders 1980). Such a response might help to maintain the infection at low densities, perhaps enhancing any reduction in host density brought about by a sterilizing parasite.

Model I shows that, when there are no competitors, sterilization of the host does not affect the capacity of a sterilizing virus to invade and persist in a well-mixed host population. Barlow (1994) came to a similar conclusion in a model of VVIC in New Zealand possums. Assuming that the sterilizing virus was sexually transmitted and that there was no recovered-and-immune stage (i.e. $v \rightarrow 0$ ), he concluded that a sterilizing virus could become established in the host population, but that the reduction in host density would be slower than could be expected for a lethal agent.

Both the competition criterion of Barlow (1994) and that developed here show that persistence of an immunocontraceptive vector in the face of competition is possible if transmissibility can be engineered into the vector, or if changes in host behaviour as a result of sterilization increase the rate of infectious contacts (Ji, Clout \& Sarre 2000). Neither scenario seems likely in the rabbit-myxoma virus system, for there are no plans to increase the inherent transmissibility of the disease, and any behavioural changes are unlikely to affect transmission of a disease that is spread mechanically on the mouthparts of insects (Fenner, Day \& Woodroofe 1956). Consequently, repeated releases will be required to establish and maintain the virus in regions where myxomatosis is already present. As epidemics of myxomatosis are driven mainly by seasonal changes in the availability of susceptible kittens and insect vectors (Wheeler \& King 1985; Parer \& Korn 1989), a sterilizing myxoma virus could be released in populations prior to the normal occurrence of the disease, thereby preempting infection with field viruses. A recent experiment, in which a myxoma virus was strategically released at four field sites, suggests that it will be feasible to introduce recombinant viruses into wild rabbit populations (Robinson et al. 1997). Moreover, Model II shows that sterilization confers no competitive disadvantage within a population, and so a sterilizing virus might persist for a long time following strategic release. This would at least allow the possibility of control of rabbits by inundative release of the sterilizing virus (sensu Jaffee 1993) if the classical biological control strategy fails.

We have assumed in this analysis that the parameters of non-sterilizing viruses are, in some sense, the result of co-evolution of the host and virus, and that there will be no evolutionary change in a sterilizing virus or the host. The pay-off therefore represents the initial return on investment from VVIC; in the longer term the pay-off can only be predicted if we can predict the outcome of co-evolutionary processes.

A question that cannot be addressed in the model presented here is how spread between populations will affect the persistence of competing strains. Barlow $(1994,1997)$ alluded to the potential importance of this process, and Sato, Matsuda \& Sasaki (1994) have shown that dispersal processes can affect population dynamics in a model that includes sterilization of infected hosts. These questions will be addressed in subsequent work.

\section{Acknowledgements}

This research was supported by an Australian postgraduate research award to G. M. Hood and by the Pest Animal Control Co-operative Research Centre. We thank two anonymous referees whose suggestions substantially improved the manuscript.

\section{References}

Anderson, R.M. \& May, R.M. (1979) Population biology of infectious diseases. Part I. Nature, 280, 361-367.

Anderson, R.M. \& May, R.M. (1981) The population dynamics of microparasites and their invertebrate hosts. Philosophical Transactions of the Royal Society of London, Series B, 291, 451-524.

Anderson, R.M. \& May, R.M. (1982) Coevolution of hosts and parasites. Parasitology, 85, 411-426.

Anderson, R.M. \& May, R.M. (1991) Infectious Diseases of Humans: Dynamics and Control. Oxford University Press, Oxford, UK.

Atkinson, I.A.E. (1989) Introduced animals and extinctions. Conservation for the Twenty-First Century (eds D. Western \& M.C. Pearl), pp. 54-75. Oxford University Press, Oxford, UK. 
Biological control using sterilizing

viruses
Barlow, N.D. (1994) Predicting the effect of a novel vertebrate biocontrol agent: a model for viral-vectored immunocontraception of New Zealand possums. Journal of Applied Ecology, 31, 454-462.

Barlow, N.D. (1997) Modelling immunocontraception in disseminating systems. Reproduction, Fertility and Development, 9, 51-60.

Barlow, N.D. \& Kean, J.M. (1998) Simple models for the impact of rabbit calicivirus disease (RCD) on Australasian rabbits. Ecological Modelling, 109, 225-241.

Barlow, N.D., Kean, J.M. \& Briggs, C.J. (1997) Modelling the relative efficacy of culling and sterilisation for controlling populations. Wildlife Research, 24, 129-141.

Beddington, J.R., Free, C.A. \& Lawton, J.H. (1978) Characteristics of successful natural enemies in models of biological control. Nature, 273, 513-519.

Bremerman, H.J. \& Thieme, H.R. (1989) A competitive exclusion principle for pathogen virulence. Journal of Mathematical Biology, 27, 179-190.

Briggs, C.J. \& Godfray, H.C.J. (1995) The dynamics of insect-pathogen interactions in stage-structured populations. American Naturalist, 145, 855-887.

Briggs, C.J. \& Godfray, H.C.J. (1996) The dynamics of insect-pathogen interactions in seasonal environments. Theoretical Population Biology, 50, 149-177.

Busenberg, S.N. \& Hadeler, K.P. (1990) Demography and epidemics. Mathematical Biosciences, 101, 63-74.

Chambers, L.K., Singleton, G.R. \& Hood, G.M. (1997) Immunocontraception as a potential control method of wild rodent populations. Belgian Journal of Zoology, 127, 145-156.

Chesson, P. (1994) Multispecies competition in variable environments. Theoretical Population Biology, 45, 227276.

Chesson, P. (1997) Making sense of spatial models in ecology. Modeling Spatiotemporal Dynamics in Ecology (eds J. Bascompte \& R.V. Solé), pp. 151-166. Springer-Verlag/Landes Bioscience, New York, NY.

Chesson, P. \& Huntly, N. (1997) The roles of harsh and fluctuating conditions in the dynamics of ecological communities. American Naturalist, 150, 519-553.

Coleman, J.D. (1993) The integration of management of vertebrate pests in New Zealand. New Zealand Journal of Zoology, 20, 341-345.

Cooke, B.D. (1997) Analysis of the Spread of Rabbit Calicivirus from Wardang Island Through Mainland Australia. Project CS.236. Australian Meat Research Corporation, Canberra, Australian Capital Territory.

Cooke, K.L. \& Vandendriessche, P. (1996) Analysis of an SEIRS epidemic model with two delays. Journal of Mathematical Biology, 35, 240-260.

Courchamp, F. \& Cornell, S. (2000) Virus-vectored immunocontraception to control feral cats on islands: a mathematical model. Journal of Applied Ecology, 37, 903-913.

Courchamp, F. \& Sugihara, G. (1999) Modeling the biological control of an alien predator to protect island species from extinction. Ecological Applications, 9, 112123.

Cowan, P.E. \& Tyndale-Biscoe, C.H. (1997) Australian and New Zealand mammal species considered to be pests or problems. Reproduction, Fertility and Development, 9, 27-36.

(C) 2000 British Ecological Society Journal of Applied Ecology, 37, 914-925 pp. 3-16. Cambridge University Press, Cambridge, UK.
Dwyer, G., Levin, S.A. \& Buttel, L. (1990) A simulation model of the population dynamics and evolution of myxomatosis. Ecological Monographs, 60, 423-448.

Edmonds, J.W., Shepherd, R.C. \& Nolan, I.F. (1978) Myxomatosis: the occurrence of antibody to a soluble antigen of myxoma virus in wild rabbits, Oryctolagus cuniculus (L.), in Victoria, Australia. Journal of Hygiene (London), 81, 245-249.

Fenner, F. \& Ratcliffe, F.N. (1965) Myxomatosis. Cambridge University Press, Cambridge, UK.

Fenner, F. \& Ross, J. (1994) Myxomatosis. The European Rabbit: The History and Biology of a Successful Colonizer (eds H.V. Thompson \& C.M. King), pp. 205-239. Oxford University Press, Oxford, UK.

Fenner, F., Day, M.F. \& Woodroofe, G.M. (1956) The epidemiological consequences of the mechanical transmission of myxomatosis by mosquitoes. Journal of Hygiene (Cambridge), 54, 284-303.

Ferriere, R. \& Gatto, M. (1995) Lyapunov exponents and the mathematics of invasion in oscillatory or chaotic populations. Theoretical Population Biology, 48, 126171.

Fountain, S., Holland, M.K., Hinds, L.A., Janssens, P.A. \& Kerr, P.J. (1997) Interstitial orchitis with impaired steroidogenesis and spermatogenesis in the testes of rabbits infected with an attenuated strain of myxoma virus. Journal of Reproduction and Fertility, 110, 161169.

Gilbert, N., Myers, K., Cooke, B.D., Dunsmore, J.D., Fullagar, P.J., Gibb, J.A., King, D.R., Parer, I., Wheeler, S.H. \& Wood, D.H. (1987) Comparative dynamics of Australasian rabbit populations. Australian Wildlife Research, 14, 491-503.

Hethcote, H.W. \& van den Dreissche, P. (1991) Some epidemiological models with non-linear incidence. Journal of Mathematical Biology, 25, 359-380.

Holland, M.K. \& Jackson, R.J. (1994) Virus-vectored immunocontraception for control of wild rabbits: identification of target antigens and construction of recombinant viruses. Reproduction, Fertility and Development, 6, 631-642.

Hone, J. (1992) Rate of increase and fertility control. Journal of Applied Ecology, 29, 695-698.

Hone, J. (1999) On rate of increase $(r)$ : patterns of variation in Australian mammals and the implications for wildlife management. Journal of Applied Ecology, 36, 709-718.

Hood, G.M. (2000) Spatial host-parasite models: application to biological control of the European rabbit (Oryctolagus cuniculus L.) using immunocontraceptive viruses. PhD Thesis. Australian National University, Australia, Canberra, Australian Capital Territory.

Jaffee, B.A. (1993) Density-dependent parasitism in biological control of soil-borne insects, nematodes, fungi and bacteria. Biocontrol Science and Technology, 3, 235-246.

Ji, W., Clout, M.N. \& Sarre, S.D. (2000) Biological control of brushtail possums through sterilisation: responses of male possums to sterile females. Journal of Applied Ecology, 37, 926-934.

Kerr, P.J., Twigg, L.E., Silvers, L., Lowe, T.J. \& Forrester, R.I. (1998) Serological monitoring of the epidemiology of myxoma virus to assess the effects of imposed fertility control of female rabbits on myxomatosis. Wildlife Research, 25, 123-131.

Levin, S.A. \& Pimental, D. (1981) Selection of intermediate rates of increase in parasite-host systems. American Naturalist, 117, 308-315.

Liu, W., Hethcote, H. \& Levin, S.A. (1987) Dynamical behaviour of epidemiogical models with nonlinear inci- 
G.M. Hood,

$P$. Chesson \&

R.P. Pech dence rates. Journal of Mathematical Biology, 25, 359380.

Liu, W., Levin, S.A. \& Iwasa, Y. (1986) Influence of nonlinear incidence rates upon the behaviour of SIRS epidemiological models. Journal of Mathematical Biology, 23, 187-204.

McCallum, H. (1996) Immunocontraception for wildlife population control. Trends in Ecology and Evolution, 11, 491-493.

Massad, E. (1987) Transmission rates and the evolution of pathogenicity. Evolution, 41, 1127-1130.

May, R.M. \& Anderson, R.M. (1983) Epidemiology and genetics in the coevolution of parasites and hosts. Proceedings of the Royal Society of London, Series B, 219, 281-313.

May, R.M. \& Nowak, M.A. (1994) Superinfection, metapopulation dynamics, and the evolution of diversity. Journal of Theoretical Biology, 170, 95-114.

Mena-Lorca, J. \& Hethcote, H.W. (1992) Dynamic models of infectious diseases as regulators of population sizes. Journal of Mathematical Biology, 30, 693-716.

Muller, L.I., Warren, R.J. \& Evans, D.L. (1997) Theory and practice of immunocontraception in wild mammals. Wildlife Society Bulletin, 25, 504-514.

Myers, K., Parer, I., Wood, D. \& Cooke, B. (1994) The rabbit in Australia. The European Rabbit: The History and Biology of a Successful Colonizer (eds H.V. Thompson \& C.M. King), pp. 108-157. Oxford University Press, Oxford, UK.

Mykytowycz, R. (1961) Social behaviour of an experimental colony of wild rabbits, Oryctolagus cuniculus (L.). IV. Conclusion: outbreak of myxomatosis, third breeding season, and starvation. CSIRO Wildlife Research, 6, $142-155$.

Mykytowycz, R. \& Fullagar, P.J. (1973) Effect of social environment on reproduction in the rabbit, Oryctolagus cuniculus (L.). Journal of Reproduction and Fertility Supplement, 19, 503-522.

Nowak, M.A. \& May, R.M. (1994) Superinfection and the evolution of parasite virulence. Proceedings of the Royal Society of London, Series B, 255, 81-89.

Onstad, D.W. (1992) Temporal and spatial scales in epidemiological concepts. Journal of Theoretical Biology, 158, 495-515.

Parer, I. \& Korn, T.J. (1989) Seasonal incidence of myxomatosis in New South Wales. Australian Wildlife Research, 16, 563-568.

Parer, I., Sobey, W.R., Conolly, D. \& Morton, R. (1994) Virulence of strains of myxoma virus and the resistance of wild rabbits, Oryctolagus cuniculus (L.), from different locations in Australasia. Australian Journal of Zoology, 42, 347-362.

Pech, R.P. \& Hood, G.M. (1998) Foxes, rabbits, alternative prey and rabbit calicivirus disease: consequences of a new biological control agent for an outbreaking species in Australia. Journal of Applied Ecology, 35, 434- 453.

Poole, W.E. (1960) Breeding of the wild rabbit, Oryctolagus cuniculus (L.) in relation to the environment CSIRO Wildlife Research, 5, 21-43.

Robinson, A.J., Jackson, R., Kerr, P., Merchant, J., Parer, I. \& Pech, R. (1997) Progress towards using recombi- nant myxoma virus as a vector for fertility control in rabbits. Reproduction, Fertility and Development, 9, 7783.

Roizman, B. (1990) Herpesviridae: a brief introduction. Fields Virology (eds B.N. Fields, D.M. Knipe, R.M. Chanock, M.S. Hirsch \& T.P. Monath), pp. 1787-1793. Raven Press, New York, NY.

Ross, J., Tittensor, A.M., Fox, A.P. \& Sanders, M.F. (1989) Myxomatosis in farmland rabbit populations in England and Wales. Epidemiology and Infection, 103, 333-357.

Sato, K., Matsuda, H. \& Sasaki, A. (1994) Pathogen invasion and host extinction in lattice structured populations. Journal of Mathematical Biology, 32, 251-268.

Saunders, I.W. (1980) A model for myxomatosis. Mathematical Biosciences, 48, 1-15.

Seymour, R.M. (1992) A study of the interaction of virulence, resistance and resource limitation in a model of myxomatosis mediated by the European rabbit flea Spilopsyllus cuniculi (Dale). Ecological Modelling, 60, 281-308.

Smith, G.C. \& Trout, R.C. (1994) Using Leslie matrices to determine wild rabbit population growth and the potential for control. Journal of Applied Ecology, 31, 223-230.

Stenseth, N.C. (1981) How to control pest species: application of models from the theory of island biogeography in formulating pest control strategies. Journal of Applied Ecology, 18, 773-794.

Twigg, L.E., Lowe, T.J., Martin, G.R., Wheeler, A.G., Gray, G.S., Griffin, S.L., O'Reilly, C.M., Robinson, D.J. \& Hubach, P.H. (2000) Effects of surgically imposed sterility on free-ranging rabbit populations. Journal of Applied Ecology, 37, 16-39.

Tyndale-Biscoe, C.H. (1991) Fertility control in wildlife. Reproduction, Fertility and Development, 3, 339-343.

Tyndale-Biscoe, C.H. (1994) Virus-vectored immunocontraception of feral mammals. Reproduction, Fertility and Development, 6, 281-287.

Wheeler, S.H. \& King, D.R. (1985) The European rabbit Oryctolagus cuniculus in south-western Australia. III. Survival. Australian Wildlife Research, 12, 213-226.

Williams, C.K. \& Twigg, L.E. (1996) Responses of wild rabbit populations to imposed sterility. Frontiers of Population Ecology (eds R.B. Floyd, A.W. Sheppard \& P.J. De Barro), pp. 547-560. CSIRO Publishing, Melbourne, Australia.

Williams, C.K., Parer, I., Coman, B.J., Burley, J. \& Braysher, M.L. (1995) Managing Vertebrate Pests. Rabbits. Bureau of Resource Sciences/CSIRO Division of Wildlife and Ecology, Australian Government Publishing Service, Canberra, Australia.

Zhou, J.S. (1995) An SIS disease transmission model with recruitment-birth-death demographics. Mathematical and Computer Modelling, 21, 1-11.

Zhou, J.S. \& Hethcote, H.W. (1994) Population size dependent incidence in models for diseases without immunity. Journal of Mathematical Biology, 32, 809-834.

Received 24 August 1999; revision received 31 March 2000
(C) 2000 British

Ecological Society

Journal of Applied

Ecology, 37 .

914-925 